\title{
Leadership \& Professional Development: Having a Backup Plan
}

\author{
Claiborne B Childs, MD ${ }^{1,2 *}$, S Ryan Greysen, MD, MHS, MA ${ }^{1}$
}

'Section of Hospital Medicine, University of Pennsylvania Health System, Philadelphia, Pennsylvania; ${ }^{2}$ University of Pennsylvania Perelman School of Medicine, Philadelphia, Pennsylvania.

"Confidence comes from being prepared." -John Wooden

Hospital medicine is a field that requires a constant state of readiness and flexibility. With respect to patient care, constant preparedness is required because conditions change. This necessitates always having a backup plan, or Plan B. For example, your patient with a gastrointestinal (GI) bleed should have two large-bore intravenous (IV) catheters and packed red blood cells (RBCs) typed and crossed. If the patient becomes unstable, the response is not just doing more of the same (IV fluids and proton pump inhibitors); the focus shifts to your Plan B: call Gl, transfuse blood, transfer the patient to the intensive care unit.

In contrast to clinical scenarios, there is often a lack of readiness to deal with rapid changes in workflow. Without a plan, efficiency decreases, stress levels rise, and both patients and providers alike suffer the consequences. Patients spending extended periods of time in the Emergency Department (ED) receive less timely services and often don't benefit from the expertise that they would receive in inpatient units. ${ }^{1}$ This is particularly true in an era in which many hospitals are experiencing higher overall volume and surges are more common.

Ideally, readiness should manifest as the ability to adapt to changes at the individual, hospitalist team, and leadership levels. Having a Plan B in the practice of hospital medicine is a focused exercise for anticipating future problems and addressing them prospectively. When thinking about a Plan B, the following are some steps to consider:

1. Identify Triggers. In the earlier example of the Gl bleed, our triggers for Plan B would be a change in vitals or a brisk drop in hemoglobin. Regarding hospital workflow, the triggers might include low service or bed capacity or a decreased number of expected discharges for the day. Perhaps a high ED census or increased surgical volume will trigger your plan to handle the surge.

*Corresponding Author: Claiborne Bernard Childs, MD; Email: Claiborne. Childs@pennmedicine.upenn.edu; Telephone: 215-662-3797; Twitter: @DrChildsMD.

Received: March 5, 2020; Accepted: March 5, 2020

(c) 2020 Society of Hospital Medicine DOI 10.12788/jhm.3528
2. Define Your Response. At both an individual and service level, there are steps you might consider in your Plan B. On teaching services, this might mean prioritizing rounding on patients that you're expecting to discharge so they're able to leave the hospital sooner. For patients on observation status who are boarding in the ED for extended periods, there might be opportunities to safely discharge them with follow-up or even complete their work-up in the ED. There may be circumstances in which providers should exceed the usual service capacity and conditions in which it is truly unsafe to exceed that limit. If there are resources available to increase staffing, consider how to best utilize them.

3. Engage Broadly and Proactively. It is very difficult to execute a Plan B (or frankly a Plan A) without buy-in from your stakeholders. This starts with the rank and file, those on your team who will actually execute the plan. The leadership of your department or division, the ED, and nursing will also likely need to provide input. If financial resources for flexing up staff are part of your plan, the hospital administration might need to weigh in. It is best to engage stakeholders early on rather than during a crisis.

4. Constant Assessment and Improvement. Going back to our example of our patient with a Gl bleed, you're constantly reevaluating your patient to determine if your Plan B is working. Similarly, you should collect data and reassess the effectiveness of your plan. There are likely opportunities to improve it.

There are no textbook chapters or medical school lectures to prepare hospitalists for these real-world crises. Yet failing to have a Plan B is to surrender a tremendous amount of personal control in the face of chaos, to jeopardize patient care, and to ultimately forgo the opportunity to achieve a level of mastery in a field predicated on readiness.

Disclosures: The authors have nothing to disclose.

\section{Reference \\ 1. Institute of Medicine, Committee on the Future of Emergency Care in the United States Health System. Hospital-Based Emergency Care at the Breaking Point. Washington, District of Columbia: The National Academies Press; 2006.}

